\title{
Pros and cons of the gasless laparoscopic transhiatal esophagectomy for upper esophageal carcinoma
}

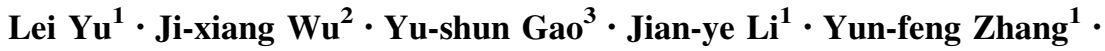 \\ $\mathrm{Ji} \mathrm{Ke} \mathrm{K}^{1}$
}

Received: 17 April 2015/Accepted: 1 August 2015/Published online: 28 September 2015

(c) The Author(s) 2015. This article is published with open access at Springerlink.com

\begin{abstract}
Background Controversies on how to treat upper esophageal carcinoma have existed for several decades. With the application of minimally invasive techniques, surgical treatment to upper esophageal carcinoma tends to show more advantages and attract more patients. Up to now, most hospitals adopted the combined thoracoscopic and laparoscopic esophagectomy (CTLE) as the way of minimally invasive surgery for upper esophageal carcinoma. But CTLE to treat upper esophageal carcinoma has its drawbacks, such as demanding certain pulmonary function and severe postoperative regurgitation. In 2011, we developed the gasless laparoscopic transhiatal esophagectomy (LTE) to treat upper esophageal carcinoma, which showed some advantages. The aim of this article was to compare LTE with CTLE in treating upper thoracic or cervical esophageal carcinoma and assess the value of LTE.
\end{abstract}

Lei $\mathrm{Yu}$

yulei1118@sohu.com

$\triangle$ Ji-xiang Wu

wjx95@hotmail.com

$\triangle$ Yu-shun Gao

1027david@sohu.com

1 Department of Thoracic Surgery, Beijing Tongren Hospital, Capital Medical University, Beijing, China

2 Department of Surgery, Beijing Tongren Hospital, Capital Medical University, No. 1 Dongjiaominxiang Street, Dongcheng District, Beijing 100730, China

3 Department of Thoracic Surgery, Cancer Institute and Hospital, Chinese Academy of Medical Sciences, Peking Union Medical College, No. 17 Panjiayuan, Chaoyang District, Beijing, China
Methods From 2009 to 2014, esophagectomy has been performed by the introduction of minimally invasive surgery in a total of 83 patients with upper thoracic or cervical esophageal carcinoma. Among these patients, LTE was performed in 27 cases (Group 1), while CTLE was performed in the other 56 (Group 2). Neoadjuvant chemotherapy was done in patients of Group 1 .

Results There were no operation-related deaths and conversion to open procedure. There was no significant difference in postoperative complications, ventilation time, ICU stay, hospital stay, and anastomotic leak rates between the two groups. But LTE was associated with shorter operative time and less intraoperative blood loss. In Group $2,21(37.5 \%)$ patients had postoperative pulmonary complications, while in Group 1, there were 6 (22.2\%) patients having pulmonary complications at least one time. Results of 24-h pH monitoring and manometry showed that postoperative laryngo-pharyngeal reflux (PLPR) was more severe in Group 2 patients than in Group 1; for Group 1, PLPR mainly occurred on sleep stage, while for Group 2, PLPR might exist all the day with short intervals and last longer at night. The median overall survival was 27.2 months after CTLE and 30.8 months after LTE $(P=0.962)$. There was no significant difference in survival at 2, 3 and 4 years between the two groups.

Conclusions Compared with CTLE, LTE is a more minimally invasive approach to effectively treat patients with upper esophageal carcinoma. Laryngo-pharyngeal reflux after LTE was less severe than that after CTLE, which might lower incidence of pulmonary complications. For the elderly patients, LTE seems more suitable.

Keywords Minimally invasive esophagectomies - Upper esophageal carcinoma $\cdot$ Outcome $\cdot$ Laryngo-pharyngeal reflux 
Controversies on how to treat upper esophageal carcinoma have existed for several decades. Some experts believed that surgery and radiotherapy to upper esophageal carcinoma could lead to equivalent results, and both of them had pros and cons [1-7]. Radiotherapy had lower rates of morbidity and mortality [1-4], while surgery might result in long-term outcome [5-7]. But with the application of minimally invasive techniques, surgical treatment to upper esophageal carcinoma tends to show more advantages and attract more patients [8, 9]. Up to now, most hospitals adopted the combined thoracoscopic and laparoscopic esophagectomy (CTLE) as the way of minimally invasive surgery for upper esophageal carcinoma. But CTLE to treat upper esophageal carcinoma has its drawbacks, such as demanding certain pulmonary function and severe postoperative regurgitation [8, 10-12]. In 2011, the gasless laparoscopic transhiatal esophagectomy (LTE) to treat upper esophageal carcinoma was developed in our department, which showed some advantages. It could be performed for the elderly or patients with severe preoperative pulmonary dysfunction. The aim of this article was to compare LTE with CTLE in treating upper thoracic or cervical esophageal carcinoma and assess the value of LTE.

\section{Materials and methods}

From 2009 to 2014, esophagectomy has been performed by the introduction of minimally invasive surgery in a total of 83 patients with upper thoracic or cervical esophageal carcinoma. Cervical esophageal carcinoma accounted for $61.4 \%$, and upper thoracic esophageal carcinoma accounted for $38.6 \%$. Among these patients, the gasless laparoscopic transhiatal technique (LTE) was performed in 27 cases (Group 1), while a combined laparoscopic and thoracoscopic technique (CTLE) was used in the other 56 (Group 2) (Table 1). Neoadjuvant chemotherapy was done in patients of Group 1. There were 13 cases in whom observed tumors obviously shrunk after neoadjuvant chemotherapy (Fig. 1). Among them, esophageal carcinoma with local advance downstaged effectively in 11 cases. LTE was done within 4 weeks after completion of neoadjuvant chemotherapy. No one received preoperative radiotherapy.

Endoscopy with biopsy was performed before surgery and histologic examination confirmed that all were squamous cell carcinoma. Computed tomography (CT scan), ultrasonography, barium esophagram and bronchoscopy were routinely undertaken on all patients to stage preoperatively and confirm the absence of contra-indications for thoracoscopy and laparoscopy. Endoscopic ultrasound (EUS) was done to obtain accurate locoregional staging in
$56.6 \%$ patients, and positron emission tomography scan (PET) data were obtained in $34.9 \%$ patients. But 7 patients could not afford the costs of EUS or PET, so we had to rely on CT to judge the tumor stage.

The study was approved by the Human Research Ethics Board of Beijing Tongren Hospital, Capital Medical University, and Cancer Institute and Hospital, Chinese Academy of Medical Sciences. Patients were warned in regard to the potential risks prior to surgery and signed consent forms if they agreed to undergo esophagectomy by minimally invasive techniques.

\section{Follow-up}

Patients with upper thoracic or cervical esophageal carcinoma received adjuvant chemotherapy after LTE or CTLE. All patients were followed up at the outpatient clinic for the first 3 months after surgery, and then in intervals of 6 months during the postoperative 5 years. In order to objectively measure postoperative reflux, postoperative 24-h pH monitoring and manometry were undertaken and documented between 6 months and 1 year after surgery.

\section{Surgical technique}

1. The combined laparoscopic and thoracoscopic technique was performed as Luketich prescribed previously [8].

2. The gasless laparoscopic transhiatal esophagectomy was performed with five upper abdominal laparoscopic incisions and a 4-cm-long left cervical incision.

Patients were intubated under general single-lumen anesthesia, and placed in a supine position with legs spread $\left(20-30^{\circ}\right)$. At the first stage, the isobaric laparoscopy using abdominal wall lifting (Mizuho Ika Kogyo CO. LTD, Japan) was established (Fig. 2). Besides a 4-cm-long midline incision below subxyphoid made to facilitate a cotton tape and a Gauze pad into the abdomen, there were other four trocars (one 10-mm trocar and three 5-mm trocars) placed. The first step is to mobilize the stomach with the ultrasonic coagulating shears. The gastrocolic omentum is divided with care taken to preserve the right gastroepiploic arcade. Special attention should be paid when dividing the short gastric vessels. The stomach is retracted inferiorly; the short gastric vessels are identified and then divided cautiously with the ultrasonic coagulating shears. Next, the gastrohepatic ligament was opened widely. The dissection then was carried up and down the right and left crura and into the lower mediastinum. The entire periphery of the abdominal esophagus was separated.

The left gastric artery and vein could be exposed from the lesser curve view and transected at its origin with the 

patients with upper esophageal cancer undergoing LTE or CTLE
Table 1 Characteristics of

\begin{tabular}{lll}
\hline & Group 1 (27 cases) & Group 2 (56 cases) \\
\hline Sex & 21 & 43 \\
Male & 6 & 13 \\
Female & $72(47-89)$ & $61(41-78)$ \\
Median age (range) & & \\
Tumor site & 19 & 31 \\
Cervical esophagus & 8 & 25 \\
Upper thoracic esophagus. & & \\
Stage & 6 & 9 \\
I & 9 & 32 \\
II & 12 & 15 \\
III & $131 \pm 29$ & $175 \pm 15$ \\
Mean operative time (minute) & $189 \pm 52$ & $336 \pm 87$ \\
Intraoperative blood loss (ml) & Squamous cell carcinoma & Squamous cell carcinoma \\
Histologic type & $7(3-18)$ & $18(11-26)$ \\
No. of lymph nodes dissected & $1(0-5)$ & $2(0-6)$ \\
Ventilation time—days (range) & $1(0-6)$ & $2(0-7)$ \\
ICU stay—days (range) & $12(11-27)$ & $13(11-33)$ \\
Hospital stay—days (range) & & \\
\hline
\end{tabular}
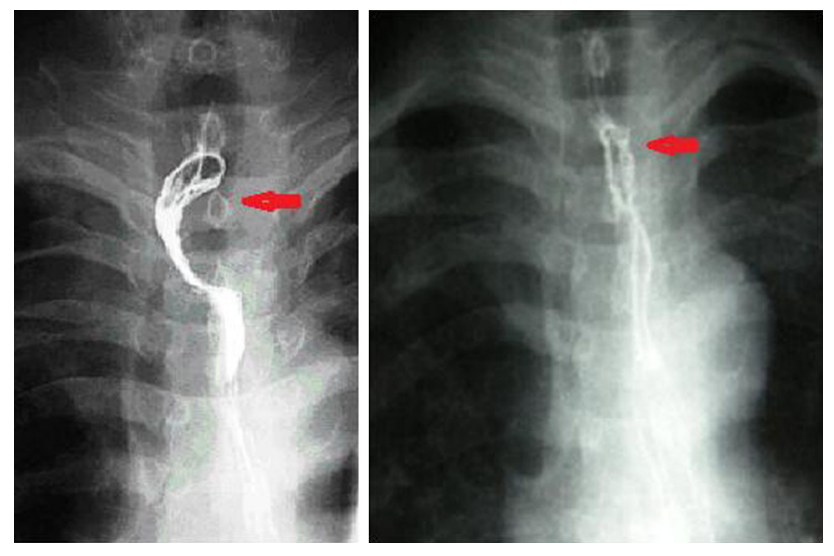

Fig. 1 Upper esophageal carcinoma with local advance downstaged effectively after neoadjuvant chemotherapy (the right picture was taken before neoadjuvant chemotherapy; the left one after neoadjuvant chemotherapy)

Endo-GIA vascular stapler. A 5-cm-wide gastric tube was constructed by firing a linear stapler 3-4 times along the greater curve of the stomach from the angle at the lesser curve to the top of the fundus.

After left-sided mobilization of the cervical esophagus, the intrathoracic normal esophagus was bluntly resected from the neck to the upper mediastinum.

At the same time, the lower esophagus was transected at the gastro-esophageal junction with the ultrasonic coagulating shears. A cotton tape was sutured to the nasogastric tube and was pulled up through the mediastinal esophagus to the neck. At the transected gastro-esophageal junction,

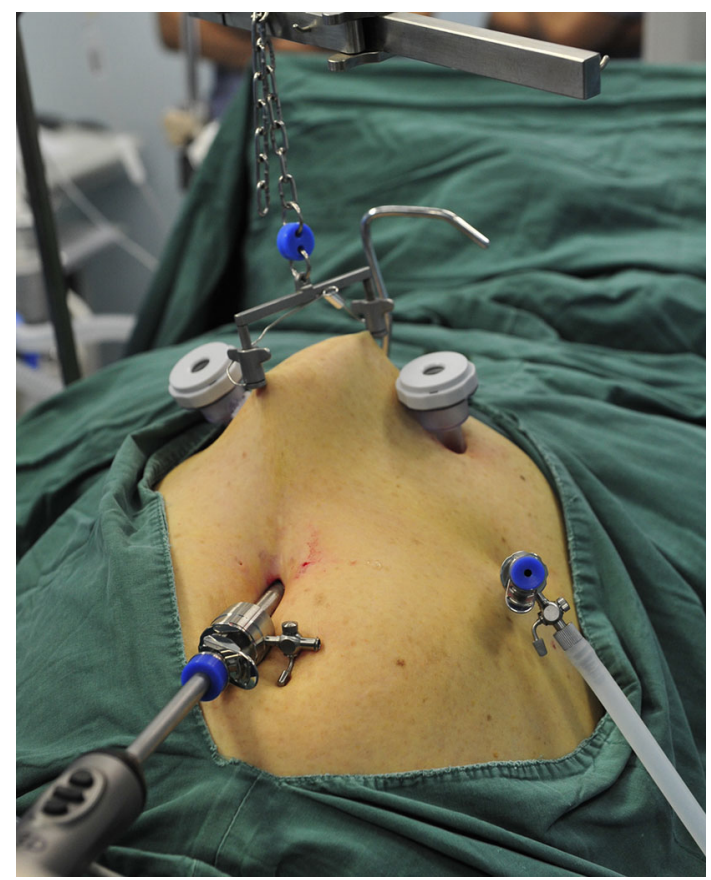

Fig. 2 Isobaric laparoscopy using abdominal wall lifting was established

the cotton tape was attached to the distal esophagus by two stitches. A surgeon held traction to the cotton tape and pulled distal esophagus up through the posterior mediastinum to the neck, followed by a Gauze pad to press the periesophageal plane. Meanwhile, the reconstructed gastric tube was stitched to the cotton tape and 3-5 min later was 
drawn up through the posterior mediastinum to the neck. Esophagogastrostomy was performed in the neck, with cervical lymphadenectomy.

\section{Endpoint}

Primary endpoints were as follows: overall survival (OS: defined as the time interval between surgery and all deaths) and postoperative laryngo-pharyngeal reflux (PLPR) (which may affect quality of life).

Secondary endpoints were as follows: the major postoperative morbidity rate (major complications occurring within 30 postoperative days and during follow-up, respectively).

\section{Statistical analyses}

Statistical analysis was performed using the Statistical Package for the Social Sciences (SPSS, ver. 13.0). All continuous data are expressed as a mean \pm standard deviation. The impact of surgery was estimated in univariate analysis. Comparisons were performed on patients with surgical treatment. Where applicable, Chi-square and Student's $t$ tests were used; survival was measured from the day of the operation until death or the last follow-up visit. Kaplan-Meier survival curves were used to compare different survival between the two groups. $P$ values less than 0.05 were considered significant.

\section{Results}

Esophagectomy by minimally invasive surgery has been successfully completed in all patients. There were no operation-related deaths and conversion to open procedure. The margin of resection was clear of tumor in all patients. Mean operative time was $131 \pm 29$ min in Group 1, while it was $175 \pm 15 \mathrm{~min}$ in Group $2(P=0.005)$. Mean intraoperative blood loss in Group 1 was $189 \pm 52 \mathrm{ml}$, significantly less than $336 \pm 87 \mathrm{ml}$ in Group $2(P=0.001)$ as shown in Table 1. There was no significant difference in postoperative complications, ventilation time, ICU stay, and hospital stay between the two groups. Within 1 month after LTE, there were 7 cases having postoperative complications (Table 2): Anastomotic leakage occurred in 3 $(11.1 \%)$ cases, pulmonary complications in $2(7.4 \%)$ case, cardiac complications in $2(7.4 \%)$ cases and herniation of part of the colon into the right thorax in one case $(3.7 \%), 4$ patients received postoperative auxiliary radiotherapy; within 1 month after CTLE, postoperative complications happened in 23 patients (Table 2): Anastomotic leakage occurred in $8(14.3 \%)$ cases, pulmonary complications in $8(14.3 \%)$, cardiac complications in 7 (12.5\%) and vocal-cord paralysis in one case $(2.8 \%)$, and nine patients received postoperative auxiliary radiotherapy. All the cases with anastomotic leakage were successfully managed within 30 days after surgery.

Follow-up was complete for all patients. Patients in Group 1 were followed up for 1-4 years, while the followup period in Group 2 was 1-5.5 years. The median diseasefree survival was 30.2 months in Group 1 and 26.4 months in Group $2(P=0.933)$. For Group 1 and Group 2, recurrence occurred in $48.1 \%(13 / 27)$ and $51.8 \%(29 / 56)$ of patients, respectively $(P=0.756)$. Survival analysis by Kaplan-Meier's method showed no statistically significant difference between Group 1 and Group 2. The curves for disease-free (Fig. 3) and overall survival (Fig. 4) were similar after surgery without a difference favoring one technique over the other. The median overall survival was 27.2 months after the CTLE and 30.8 months after the laparoscopic transhiatal esophagectomy $(P=0.962)$. 2-, 3and 4-year survival rates in Group 1 were 56.2, 42.5 and $32.1 \%$, respectively, While in Group 2, 2-, 3-, 4- and 5-year survival rates were $61.9,34.9,29.2$ and $19.5 \%$, respectively. There was no significant difference in survival at 2, 3 and 4 years between the two groups. In Group 1 , among 11 deaths, eight were associated with recurrence or metastasis and three were due to heart failure. In Group 2, 23 patients died of recurrence or metastasis, three deaths were attributable to pulmonary complications, two died of portal vein thrombosis, and one death was related to heart failure.

All the patients complained about heartburn and regurgitation from time to time after surgery. Major complications more than 6 months after surgery were pneumonia and anastomotic stricture (Table 2): in Group 1, there were 4 patients having pulmonary complications at least one time and 4 patients experiencing anastomotic stricture, while in Group 2, 13 patients had postoperative pulmonary complications $(P=0.374)$ and seven had postoperative anastomotic stricture $(P=0.771)$. Anastomotic stricture was managed successful by anastomotic dilatations.

Results of postoperative 24-h $\mathrm{pH}$ monitoring and manometry were documented as shown in Table 3. Maybe due to short length of residual esophagus, no consistent motility pattern was detected. PLPR was more severe in Group 2 than in Group 1: By univariate analysis, the total number of reflux events $(\mathrm{pH}<4)$, the number of reflux episodes (lasting $>5 \mathrm{~min}$ ) and the reflux time in Group 1 were less than those in Group $2(P=0.00)$, while the longest episode of reflux in Group 1 was longer than that in Group $2(P=0.01)$. 24-hour ambulatory esophageal $\mathrm{pH}$ monitoring also revealed that for patients in Group 1, PLPR mainly occurred at sleep stage, while for patients in Group 2, PLPR might exist with short intervals all day and last longer at night (Fig. 5). 
Table 2 Comparisons of postoperative complications between the two groups

\begin{tabular}{llll}
\hline & Group 1 (27 cases) & Group 2 (56 cases) & $P$ Value \\
\hline Complications 1 month after surgery & 7 & 23 & 0.178 \\
Pulmonary complications & 2 & 8 & \\
Anastomotic leakage & 3 & 8 \\
Cardiac complications & 2 & 7 & \\
Vocal-cord paralysis & 0 & 1 & \\
herniation & 1 & 0 & \\
Wound infection & 1 & 4 & 0.374 \\
Pulmonary complications 6 months after surgery & 4 & 13 & 0.771 \\
Anastomotic stricture & 4 & 7 & \\
\hline
\end{tabular}

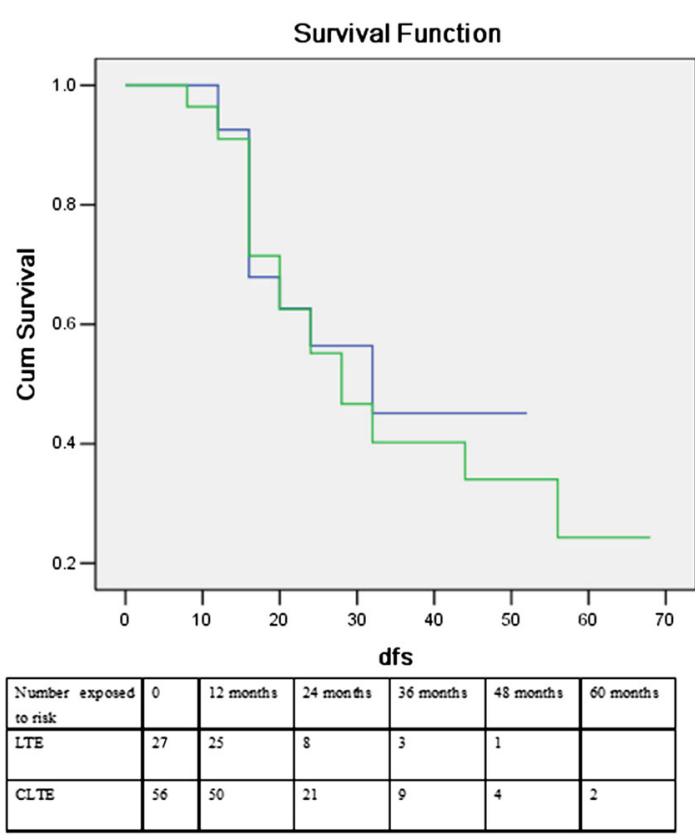

Fig. 3 Disease-free survival curves between patients undergoing LTE and CTLE

\section{Discussion}

Up to now, esophagectomy with curative intent has been regarded as the most effective treatment for early-stage esophageal carcinoma [5-7]. Conventional approaches, involving laparotomy and/or thoracotomy, are associated with high incidence of morbidities, which delay the recovery. Especially for upper esophageal carcinoma, cervicothoracoabdominal esophagectomy is one of the most complex surgical procedures with great trauma, making some surgeons and patients dismay.

A great improvement in esophagectomy is the application of minimally invasive techniques. Since Swanstrom and Hansen introduced their experience on a completely laparoscopic approach to esophageal cancer in 1997 [13],

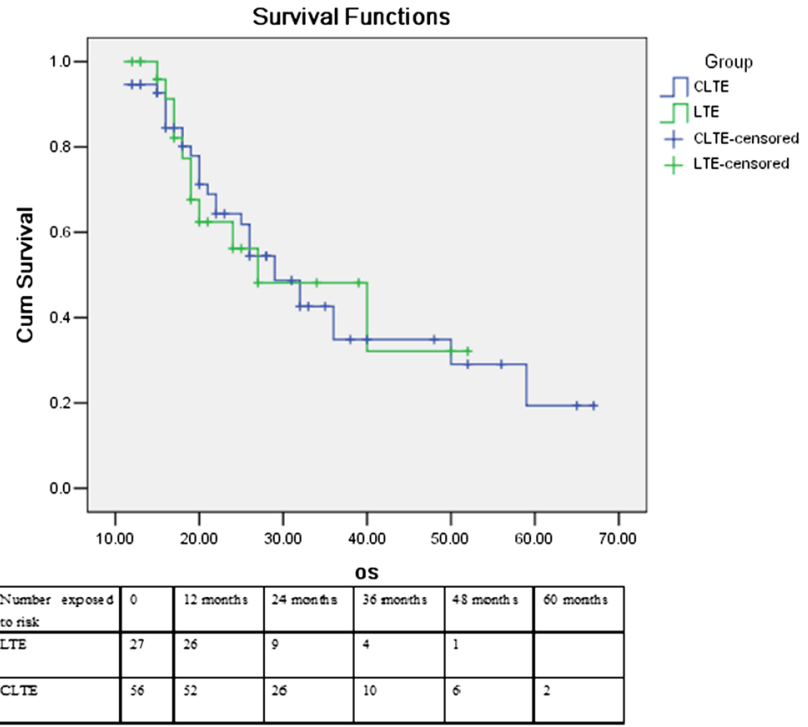

Fig. 4 Kaplan-Meier curves showing overall survival between patients undergoing LTE and CTLE

the application of minimally invasive surgery for esophageal cancer has become rapidly widespread in recent years.

CTLE has been performed in our department in 2009, and it showed significant advantages over open procedure in mortality and morbidity as other studies reported [14]. Due to the use of advanced instruments, current minimally invasive esophagectomies hold normal laparoscopic or thoracoscopic advantages. Both CTLE and LTE were associated with short period of ICU and hospital stay, relatively quick recovery, small incisions, and so on.

But in recent years, the aging demographics and the growing number of the elderly patients with upper esophageal carcinoma were becoming surgical problems we faced. Could we develop other minimally invasive techniques for upper esophageal carcinoma to decrease early postoperative risk? Can we develop some kind of more minimally invasive esophagectomy which the elderly or patients with severe preoperative pulmonary dysfunction 
Table 3 Multiple comparisons of 24-h pH monitoring and manometry (more than 6 months after surgery) between Group 1 and Group 2: $(\bar{x} \pm s)$

\begin{tabular}{lccc}
\hline & Group 1 (21 cases) & Group 2 (45 cases) & $P$ value \\
\hline Total number of reflux events $(\mathrm{pH}<4)$ & $15.33 \pm 2.82$ & $28.76 \pm 4.57$ & 0.001 \\
Number of reflux episodes (lasting $>5 \mathrm{~min})$ & $5.19 \pm 1.57$ & $15.07 \pm 1.85$ & 0.000 \\
The reflux time & $2.28 \pm 0.59$ & $5.02 \pm 0.50$ & 0.000 \\
The longest episode of reflux (min) & $29.50 \pm 4.83$ & $22.51 \pm 3.09$ & 0.001 \\
UESP (mm Hg) & $12.34 \pm 1.35$ & $11.97 \pm 1.00$ & 0.217 \\
UESL $(\mathrm{cm})$ & $1.88 \pm 0.46$ & $1.75 \pm 0.34$ & 0.227 \\
\hline
\end{tabular}

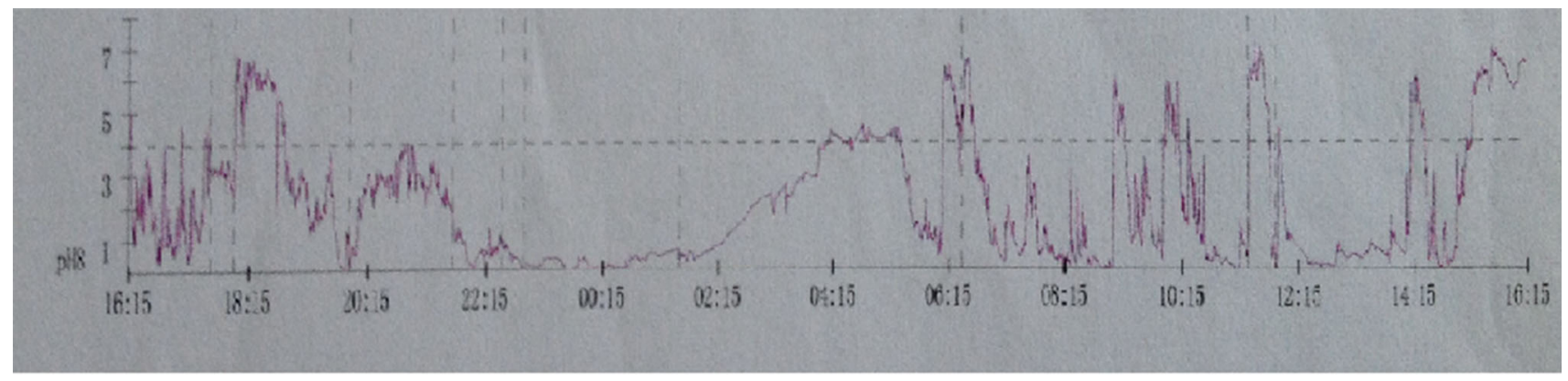

PLPR might exist with short intervals all day and last longer at night after CLTE

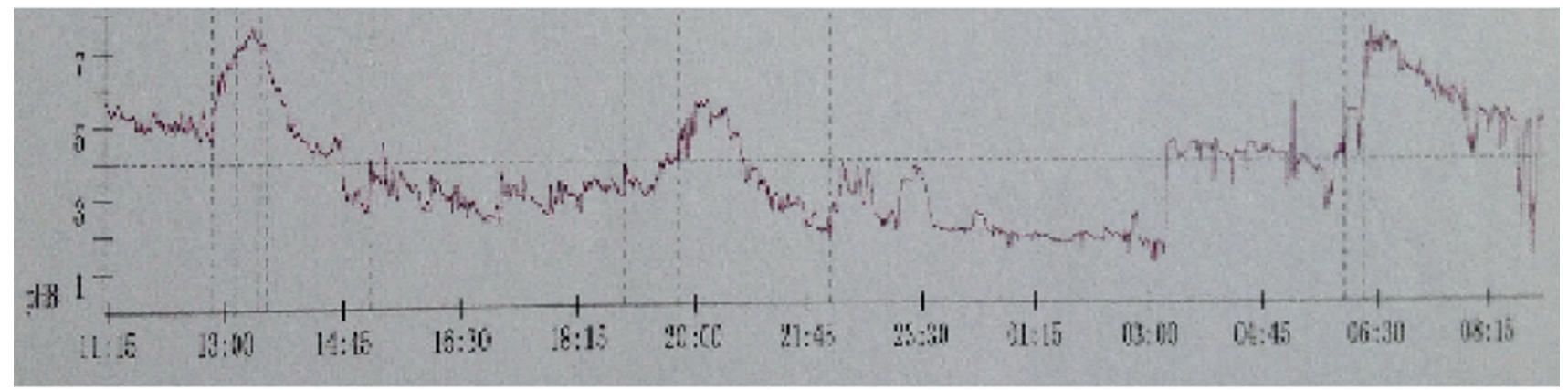

PLPR mainly occurred on sleep stage after LTE

Fig. 5 Comparison of postoperative 24-h pH monitoring 6 months after CTLE or LTE

could withstand? Would neoadjuvant chemotherapy be helpful to improve long-term outcome of minimally invasive esophagectomy without formal lymphadenectomy? Based on the questions mentioned above, the gasless laparoscopic transhiatal esophagectomy was developed.

Compared with CTLE, LTE had showed its own advantages. First of all, the adverse effects of $\mathrm{CO} 2$ insufflation and single-lung ventilation are eliminated. As life expectancy rises in China, many patients 80 years old and higher with esophageal cancer expect to prolong their life by surgical treatment. These patients do not have adequate pulmonary reserve [15], so it seems difficult for them to tolerate pneumoperitoneum and single-lung ventilation. The procedure of LTE has little to do with the thoracic cavity and lung. So poor pulmonary function was no obstacle to surgical treatment to upper esophageal carcinoma any more. In Group 1, eight patients with low pulmonary function (80 years old or greater) successfully underwent LTE. LTE can be safely performed for upper esophageal carcinoma with severe preoperative pulmonary dysfunction. Secondly, the peritoneal cavity does not need to be sealed airtight when performing LTE. This facilitates several steps of the procedure. The operating time is decreased because an optimal view can be maintained even during irrigation suction. In our series, mean operative time in Group 1 was only $131 \pm 29 \mathrm{~min}$, significantly lower than that in Group $2(P=0.000)$. What is more, benefits of LTE were also linked with a lower mean intraoperative blood loss. Mean intraoperative blood loss of LTE was only $189 \pm 52 \mathrm{ml}$, even significantly lower than CTLE $(P=0.001)$. Finally, severe PLPR happened less in Group 1 than that in Group 2. PLPR occurred every day in 
patients undergoing CTLE and LTE. PLPR had a major effect on quality of life of patients experiencing esophagectomy. High incidence of postoperative pulmonary complications was mainly related to severe PLPR. Tracking PLPR can provide important clues as to postoperative pulmonary complication and factors influencing postoperative quality of life [16]. It should be noted that in our series, incidence rates of postoperative pulmonary complications, including those occurring within 30 postoperative days and during follow-up, are relatively high. In Group 2, $21(37.5 \%)$ patients had postoperative pulmonary complications, while in Group 1, there were six (22.2\%) patients having pulmonary complications at least one time $(P=0.164)$. All the patients experiencing CTLE or LTE complained about heartburn and regurgitation from time to time. In Group 2, it was more severe than in Group 1. In Group 1, PLPR mainly occurred at sleep stage, while for patients in Group 2, PLPR might exist all the day only with short intervals and last longer at night. This might lead to higher incidence of postoperative pulmonary complications in Group 2. Mediastinal tissue press may contribute to reducing occurrence of PLPR and postoperative pulmonary complications in patients undergoing LTE (Fig. 6).

For a long period of time, the transhiatal esophagectomy has been regarded as a controversial procedure because of failure to do extensive lymphadenectomy. In order to try to decrease the possible influence of inadequate lymph-node dissection, patients undergoing LTE in this study received neoadjuvant and adjuvant chemotherapy. It is uncertain whether neoadjuvant chemotherapy followed by esophagectomy might lead to greater long-term survival [17-19]. But survival analysis by Kaplan-Meier's method demonstrated that overall survival and disease-free survival in Group 1 seem to be similar to those in Group 2. The median overall survival of Group 1 and Group 2 reached up to 30.8 and 27.2 months, respectively $(P=0.962)$. Furthermore, neoadjuvant chemotherapy effectively
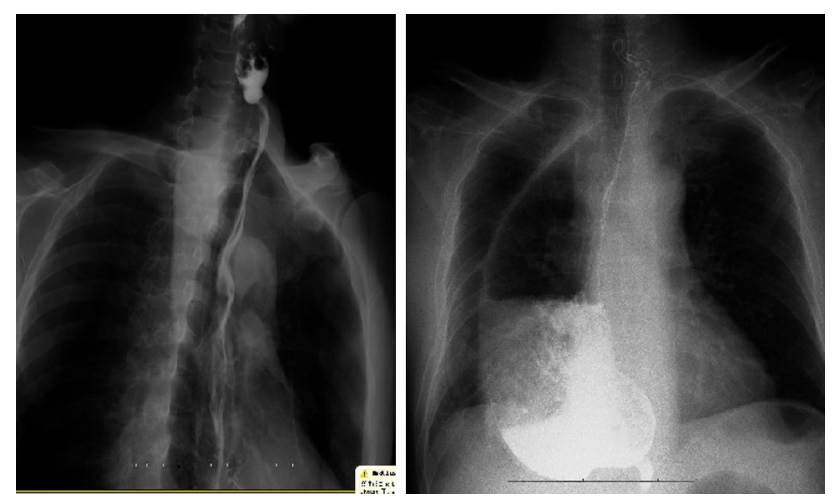

Fig. 6 Barium esophagram showed that the constructed gastric tube was limited in the posterior mediastinum 6 months after LTE (left), while it might expand in the right plural cavity 6 months after CTLE (right) downstaging esophageal carcinoma with local advance was observed in 11 cases. Downstaging these tumors made them more resectable.

In conclusion, compared with CTLE, LTE is a more minimally invasive approach to effectively treat patients with upper esophageal carcinoma. Laryngo-pharyngeal reflux after LTE was less severe than that after CTLE, which might lower incidence of pulmonary complications. For the elderly patients, LTE seems more suitable.

Acknowledgments Dr. Ji-xiang Wu, Dr. Lei Yu and Dr. Yu-shun Gao had full access to all of the data in the study and took responsibility for the integrity of the data and the accuracy of the data analysis. Dr. Ji-xiang Wu, Dr. Lei Yu, Dr. Yu-shun Gao and Dr. Jianye Li contributed substantially to the study design; Dr. Yun-feng Zhang and Dr. Ji Ke contributed mainly to data analysis and interpretation; Dr. Lei Yu and Dr. Ji-xiang Wu contributed to the writing of the manuscript. This article was corrected by Dr. Frank C. Detterbeck, Chief of the Division of Thoracic Surgery, Yale University School of Medicine.

\section{Compliance with ethical standards}

Disclosures Drs. Lei Yu, Ji-xiang Wu, Yu-shun Gao, Jian-ye Li, Yun-feng Zhang and Ji Ke have no conflicts of interest or financial ties to disclose.

Open Access This article is distributed under the terms of the Creative Commons Attribution 4.0 International License (http://creative commons.org/licenses/by/4.0/), which permits unrestricted use, distribution, and reproduction in any medium, provided you give appropriate credit to the original author(s) and the source, provide a link to the Creative Commons license, and indicate if changes were made.

\section{References}

1. Ma JB, Song YP, Yu JM et al (2011) Feasibility of involved-field conformal radiotherapy for cervical and upper-thoracic esophageal cancer. Onkologie 34(11):599-604

2. Chou SH, Li HP, Lee JY et al (2010) Radical resection or chemoradiotherapy for cervical esophageal cancer? World J Surg 34(8):1832-1839

3. Yamada K, Murakami M, Okamoto Y et al (2006) Treatment results of radiotherapy for carcinoma of the cervical esophagus. Acta Oncol 45(8):1120-1125

4. Mendenhall WM, Sombeck MD, Parsons JT et al (1994) Management of cervical esophageal carcinoma. Semin Radiat Oncol 4(3):179-191

5. Tong DK, Law S, Kwong DL et al (2011) Current management of cervical esophageal cancer. World J Surg 35(3):600-607

6. Lei D, Pan X, Luan X et al (2002) Surgical management of cervical esophageal carcinoma. Zhonghua Er Bi Yan Hou Ke Za Zhi 37(2):86-89

7. Wang WG, Li JD, Qi JX et al (2008) Experience of surgical treatment for cervical esophageal carcinoma. Zhonghua Wei Chang Wai Ke Za Zhi 11(1):19-23

8. Luketich JD, Alvelo-Rivera M, Buenaventura PO, Christie NA et al (2003) Minimally invasive esophagectomy: outcomes in 222 patients. Ann Surg 238(4):486-494

9. Briez N, Piessen G, Bonnetain F et al (2011) Open versus laparoscopically-assisted oesophagectomy for cancer: a 
multicentre randomised controlled phase III trial-the MIRO trial. BMC Cancer 11:310

10. Gockel I, Heckhoff S, Messow CM, Kneist W, Junginger T (2005) Transhiatal and transthoracic resection in adenocarcinoma of the esophagus: does the operative approach have an influence on the long-term prognosis? World J Surg Oncol 3:40

11. Hulscher JB, van Sandick JW, de Boer AG et al (2002) Extended transthoracic resection compared with limited transhiatal resection for adenocarcinoma of the esophagus. N Engl J Med 347:1662-1669

12. Law S, Wong J (2002) Use of minimally invasive oesophagectomy for cancer of the oesophagus. Lancet Oncol 3:215-222

13. Swanstrom LL, Hansen P (1997) Laparoscopic total esophagectomy. Arch Surg 132:943-947

14. Sundaram A, Geronimo JC, Willer BL, Hoshino M, Torgersen Z, Juhasz A et al (2012) Survival and quality of life after minimally invasive esophagectomy: a single-surgeon experience. Surg Endosc 26:168-176
15. Tapias Luis F, Muniappan Ashok, Wright Cameron D et al (2013) Short and long-term outcomes after esophagectomy for cancer in elderly patients. Ann Thorac Surg 95:1741-1748

16. Koh P, Turnbull G, Attia E, LeBrun P, Casson AG (2004) Functional assessment of the cervical esophagus after gastric transposition and cervicalesophagogastrostomy. Eur J Cardiothorac Surg 25(4):480-485

17. Suzuki G, Yamazaki H, Ogo E et al (2014) Multimodal approach for cervical esophageal carcinoma: role of neoadjuvant chemotherapy. Anticancer Res 34(4):1989-1992

18. Warner S, Chang YH, Paripati H et al (2014) Outcomes of minimally invasive esophagectomy in esophageal cancer after neoadjuvant chemoradiotherapy. Ann Thorac Surg 97(2):439-445

19. Malaisrie SC, Untch B, Aranha GV et al (2004) Neoadjuvant chemoradiotherapy for locally advanced esophageal cancer: experience at a single institution. Arch Surg 139(5):532-538 